\title{
Cooperative messages to enhance the performance of $L 3$ vehicles approaching roadworks
}

\author{
Serio Angelo Maria Agriesti ${ }^{*}$ (D), Marco Ponti ${ }^{2}$, Giovanna Marchionni ${ }^{2}$ and Paolo Gandini ${ }^{2}$
}

\begin{abstract}
Introduction: In the near future, automated vehicles will drive on public roads together with traditional vehicles. Even though almost the whole academia agrees on that statement, the possible interferences between the two different kinds of driver are still to be analyzed and the real impacts on the traffic flow to be under-stood.

Objectives: Aim of this paper is to study one of the most likely $L 3$ automated system to be deployed on public roads in the short term: Highway Chauffeur. The analysis of this system is carried out on a roadwork scenario to assess the positive impacts arising from a joint implementation of the automated system and the C-ITS Use Case signaling the closure of a lane. In fact, the main contribution of this paper is the assessment of the possible benefits in travel times and driving regime arising from the joint implementation of the Highway Chauffeur system and of C-ITS messages, both for the vehicles equipped with both technologies and for the surrounding traffic.

Methods: The assessment is achieved through traffic simulations carried out with the VISSIM software and a Python script developed by the authors. The overall process is described and the obtained results are provided,

commented and compared to define the implementation of the C-ITS Use Case that could maximize the benefits of $L 3$ driving.

Results: These results showed how triggering the take-over maneuver in ad-vance fosters the bottleneck efficiency (the same speed values reached between 80 and 100\% Market Penetration for around $700 \mathrm{~m}$ range of the C-ITS message are reached at 50\% Market Penetration with a $1500 \mathrm{~m}$ range). Besides, an in-creased speed up to $30 \mathrm{~km} / \mathrm{h}$ at the bottleneck is recorded, depending on the mar-ket penetration and the message range. Finally, the delay upstream the roadworks entrance is reduced by $6 \%$ and arises at around $700 \mathrm{~m}$, without the need to deploy the message up to $1500 \mathrm{~m}$.

Conclusions: The paper investigates the impacts of take-over maneuvers and of automated driving while considering different operational parameters such as the message range. The results suggest all the potentialities of the Use Case while providing interesting figures that frame the trends related to the different imple-mentations. Finally, the tool developed to carry out the presented analysis is re-ported and made available so that hopefully the Use Case may be explored further and a precise impact assessment may be carried out with different prototypes of AVs and on different infrastructures.
\end{abstract}

Keywords: Highway chauffeur, L3 driving, C-roads Italy, C-ITS, Roadworks warning, Micro-simulation, COM-Interface, Automated driving, Take-over

\footnotetext{
* Correspondence: seriangelo@gmail.com

${ }^{1}$ Built Environment - Aalto Yliopisto, Otakaari 4, 02150 Espoo, Finland

Full list of author information is available at the end of the article
}

\section{Springer Open}

(c) The Author(s). 2021 Open Access This article is licensed under a Creative Commons Attribution 4.0 International License, which permits use, sharing, adaptation, distribution and reproduction in any medium or format, as long as you give appropriate credit to the original author(s) and the source, provide a link to the Creative Commons licence, and indicate if changes were made. The images or other third party material in this article are included in the article's Creative Commons licence, unless indicated otherwise in a credit line to the material. If material is not included in the article's Creative Commons licence and your intended use is not permitted by statutory regulation or exceeds the permitted use, you will need to obtain permission directly from the copyright holder. To view a copy of this licence, visit http://creativecommons.org/licenses/by/4.0/. 


\section{Introduction}

Automation is widely regarded as a disruptive force that will shape many of the aspects of our daily lives in the next decades and driving is no exception. In literature and among stakeholders, it is quite accepted that some form of automation will gradually be introduce in newer vehicles. The forces driving towards this change includes the need to tackle climate change, to increase driving safety and to improve traffic efficiency in a world that sees its population constantly increasing. While there is not yet consensus on the final degree of automation and on the path that will lead there, it is safe to assume at this point that at least the SAE level 3 (L3) will be reached and deployed in some restricted domains. The Highway Chauffeur is one of the systems falling in the L3 level of automation. The human driver is indeed allowed to exit the driving loop and to entrust the driving task to the automation as long as some boundary conditions are met. All these boundary conditions constitute the Operational Design Domain (ODD) of the system, within which the vehicle can drive by itself without having to bother the human authority on board. The potentialities of driving a fair share of the overall kilometers with the automated system engaged are many, both for the human driver on board and for the rest of the traffic (an automated vehicle should be able to drive more efficiently and in a safer way, granting benefits to the overall traffic flow [1, 2]). Nevertheless, even on highways, a certain ODD cannot be guaranteed continuously and on the whole network since both static and dynamic features along the route can cause the end of an ODD and trigger a take-over maneuver. During this maneuver, the vehicle alerts the human driver and allows him or her to take back control possibly before reaching the end of the ODD and with a time interval wide enough for the driver to regain situational awareness. As reported in Section 2, a time window ranging between 4 and 40 s can be needed. Studying the take-over transition is of upmost importance in order to understand where the benefits bound to an L3 vehicle ends and where negative impacts on traffic efficiency and safety start to arise. In fact, if the HC system perceives the need of disengagement just before the static or dynamic feature on the road, the driver can have short time to re-enter the driving loop. One of these features can be for example the presence of a roadworks. The sudden end of the ODD and an abrupt take-over warning can translate into swerving, abrupt braking, reduced situational awareness or straight into an incorrect driving behavior. One way to mitigate these unwanted effects is to exploit C-ITS systems and cooperative messages that can enhance the perception capabilities of the L3 vehicles and avoid sudden transitions through V2X communication. ${ }^{1}$

\footnotetext{
${ }^{1} \mathrm{~V} 2 \mathrm{X}$ includes both vehicle-to-vehicle, vehicle-to-infrastructure and vehicle-to-cloud communication.
}

In order to assess how L3 vehicles, C-ITSs and the takeover transitions could impact the overall traffic flow, a simpler scenario is chosen for the analysis in this paper: a static roadwork that causes the closure of the closing lane. One of the aims of the paper is thus to assess if, through the implementation of a C-ITS message, the Highway Chauffeur can enjoy benefits concerning travel times and driving regime (Traffic Efficiency) while in turn promoting a smoother traffic flow and an increased bottleneck capacity. Applying the evaluation framework derived in $[1,3$, 4], the following research questions are derived:

- Does carrying the take-over maneuver in advance to the roadworks vertical signage foster an enhancement in Traffic Efficiency across the bottleneck section?

- If the C-ITS broadcasts what is the closed lane and allows vehicles on the open one to keep on driving in the automated mode does the overall traffic flow benefits from that in terms of Traffic Efficiency?

- What are the impacts on the upstream section in terms of delay?

The paper is structured as it follows: in Section 2 a bibliographical review of scientific literature concerning take-over times and possible vehicle behaviors is carried out, in Section 3 the modeling software is presented highlighting the changes and adaptation chosen to simulate a take-over vehicle. Thus, in Section 4 the various scenarios and the different results are presented while they are compared in Section 5. Then, the benefits on Traffic Efficiency are obtained, together with the best implementation logics about the range of the cooperative message. In Section 6 the conclusions are presented, together with future research directions.

\section{Bibliographical study about take-over times and the possible L3 vehicle behaviour}

Being a transition in behavior from the vehicle to the human, the take-over maneuver, can be difficult to insert into a traffic modeling software. Still, to evaluate the case study presented in this paper, it was necessary to insert this transition in behavior within the designed scenarios. As explained in $[3,5]$ one of the main benefits of the C-ITS services is to improve situational awareness and to broadcast the needed information in advance. When these benefits meet automated driving, a jointed, increased benefit could be observed. In order to frame it, a bibliographical review about the possible take-over reactions is carried out in the following and exploited to design the Python script that allowed to simulate the take-over transition.

In [6] it is stated that "(...) there is no such thing as a single, general take-over time. Instead the take-over time 
is, within limits, specific for a particular set of situation variables (e.g., traffic density, action alternatives, HMI concepts, secondary task type, level of driver distraction) and driver variables (e.g. age and skill of the driver)". In fact, it is not possible to define a single time span that is applicable to each situation and for each human driver. Even the same human being is going to perform differently during different times depending on its own psychophysical features. Thus, aim of this Section is to identify a plausible range of take-over times that will be applied to the simulation scenarios. Still in [6], an integrated model approach to assess the driver behavior during take-over is presented. The work is especially relevant because it considers a human driver engaged in a secondary task while the L3 vehicle drives itself. [6] analyses all the phases that compose the take-over transition, take-over request, take-over time (composed by gaze on street and cognitive processing by the human driver), take-over (after which reaction is the actual driving response of the human driver).

This variability in take-over times is mentioned also in [7], in which the take-over process is divided among situation awareness and decision. In the first one both perception, comprehension and projection of future statuses are performed by the human driver while in the second one the actual reaction is formulated. Again, after the take-over takes place, the actual reaction of the human driver is performed and the driving ends its transition from highly automated to manual.

In [8], the take-over time performed in an automated vehicle with controlled longitudinal and lateral control on a highway scenario is obtained through a driving simulator study with 44 drivers. From their bibliographical review, the authors defined around $8 \mathrm{~s}$ as an interval wide enough for a take-over during a traffic jam or while driving on a highway. Thus, their main research question concerned the assessment of a 10s time interval as a safe and comfortable interval for the take-over. This work is relevant for the presented assessment because considers a similar scenario, namely a construction site with change in lane marking. The results showed that all the involved subjects were able to take-over control within the 10s timeslot from the presentation of the take-over request, the response time ranged between $1.4 \mathrm{~s}$ and 6.7 s. 10s is a value obtained also in [9], even if in this work the take-over request was triggered either at a systembased regular interval or when the human driver was found to look away from the road for more than a predefined interval. 10s was the time needed for the participants to resume control, in the following seconds the human drivers showed exaggeration in steering corrections for $10-15 \mathrm{~s}$ more. Only after $35-40$ s from the take-over request, the lateral control of the vehicle proved to be steady and adequate. This work is one of the few ones considering both longitudinal and lateral control during the take-over transition, this distinction is valuable indeed for the simulated scenarios in Section 3. In fact, in this work the hypothesized behavior of an L3 vehicle in take-over does not allow a lane change as long as a certain time interval has passed, this way of modeling the take-over reflects the first seconds during which the driver is re-assessing a minimum situational awareness, is reaching for the steering wheel and is checking the surroundings. In [10], the take-over time in not safety critical scenarios is analyzed in relation to different HMI designs and auditory outputs, still through a driving simulator. From the bibliographical review of the authors, a take-over time up to 30 s can be performed in not safety critical scenarios. It is also interesting to note that the authors refer explicitly to the possibilities granted by V2X communication to enhance environmental perception and to present in advance the takeover transition to the driver. Also in this study, the event triggering the end of the ODD and the take-over request were temporary lane markings; the take-over request was delivered 20s before said section. The performed reaction times were calculated for different steps: first gaze (ranging between $1.29 \div 1.42 \mathrm{~s}$ ), hands on the wheel $(5.66 \div 7.84 \mathrm{~s})$ and system deactivation $(8.26 \div 9.86 \mathrm{~s})$. The take-over maneuvers were thus carried out mostly in the first half of the available 20s time budget and with a sufficient level of comfort. Finally, in [11], the microsimulation software SUMO is exploited to model the take-over transition both downwards (from automation to human) and upwards (from human to automation), the study is particularly interesting because it considers thresholds for the take-over transition beyond which a Minimum Risk Maneuver is triggered by the vehicle. Moreover, the study explicitly mention roadworks as one of the road segments where a high number of take-overs should be expected and, in assessing the resulting effects, it is concluded that distributing the take-over maneuvers over a higher portion of the road could reduce the negative effects related to the needed change in headway. As in the present work, in [11] an increase of the headway is accounted for during the downward take-over transition to help the human driver safely retrieving the control. On the basis of the available bibliography, a take-over transition was modeled in VISSIM through the COM interface and a Python script. The following behavior was simulated:

- The HC vehicle receives the C-ITS messages or is made aware of the roadworks through vertical signaling. Either way, the vehicles start the take-over transition which is accomplished within the software by changing the vehicle type and, consequently, its behavior. 
- A vehicle that has Take-Over transition as vehicle type, is forbidden to perform a lane change as long as it remains in said vehicle type container, the driving behavior is still similar to the L3 one and the vehicle keeps on driving itself until the human driver is in the loop again.

- As soon as the Take-Over interval has passed (on the basis of the simulation seconds), the human driver is considered out of the initial phase ranging between 10 and $15 \mathrm{~s}$, as found from literature, and able to perform a lane change. This is simulated through another change of the vehicle type, that now is ruled by a human behavior and no more by the behavior of an automated vehicle. Moreover, through VISSIM settings, it is imposed that this vehicle type wants to perform a lane change as soon as the take-over interval has passed (which reflects the input of the C-ITS message, closure of a lane due to roadworks).

It should be highlighted that the reported literature review does not cover the whole state of the art about behavioral response to a take-over maneuver and was not in-tended to. The above references were the ones that allowed the authors to design the take-over script with the objective of assessing this particular case study. Many other take-over scenarios could and should be studied when analyzing the impact of L3 driving (e.g. weather conditions rather than safety critical scenarios such as crashes) and should focus both on the longitudinal and the lateral behavior of the semi-automated vehicles. Still, since in this case study the take-over was always triggered sufficiently in advance, a specific focus was given on the needed time window and the lane change capacities during the transitions.

\section{Modeling framework}

To analyze the impacts of the jointed implementation of L3 vehicles and the C-ITS Use Case Roadworks Warning - Closure of a Lane [12], the VISSIM micro-simulation software was selected. In this Section, the simulation parameters and the COM script employed are presented and commented, to guarantee the replicability of the results and to illustrate the choices and hypotheses made (on the basis of the bibliographical review of Section 2). The road network chosen for the simulations is a $7.5 \mathrm{~km}$ long road branch on the A22 infrastructure with no onand off-ramps. A22 is one of the stakeholders involved in the C-Roads Italy project, on which field tests concerning both the Highway Chauffeur system and C-ITS services will be carried out during 2020. Moreover, traffic data were provided by A22 and were exploited to calibrate the driving behavior of traditional traffic as reported in [13]. On the basis of Italian regulations as reported in [14], the closure of the slow lane due to Roadworks was designed as reported in Fig. 1.

Section 1 in Fig. 1 is located at $696 \mathrm{~m}$ from the lane closure and it is the first point with vertical signage signaling the roadworks. It should be highlighted that no indication about the lane closure is provided at this point: the first vertical signage reporting the roadworks layout is posed at $336 \mathrm{~m}$ from the lane closure (section 4). Without C-ITS messages, it is hypothesized that the L3 vehicles would start the take-over maneuver in section 1 , as soon as the roadworks presence is noticed by the on-board software through cameras. Without indications on the roadworks layout, in fact, L3 vehicles on both lanes should disengage L3 driving because they miss the information needed to assess their capability in performing the needed maneuvers (e.g. lane changes) and passing through the roadworks. If the cooperative



Fig. 1 Modeling layout 
information is received at section 1 or upstream and contains the roadworks layout, instead, only the L3 vehicles on the closing lane should disengage. The following hypotheses are made:

- The L3 vehicles are the only ones able to receive the C-ITS message; no traditional vehicle receives the information about the lane closure downstream.

- In both the C-ITS scenarios (range $696 \mathrm{~m}$ and $1500 \mathrm{~m}$ ), the L3 vehicles on the open lane are able to keep driving because, to enter the roadwork, no lane change is required. From section 1 until the start of the roadworks, these vehicles will not enter the closing lane.

- In both the C-ITS scenarios, the L3 vehicles on the closing lane receives the message and start the take-over maneuver, in order to re-engage the human driver. How this take-over transition is managed through the software is explained in the following.

- The vehicles unequipped for the reception of cooperative messages discover about the lane closure when reaching section $4,336 \mathrm{~m}$ ahead the lane closure. This reflects the vertical signals ahead a roadwork designed in the simulation.

It should be highlighted that the lane change is not completely forbidden to L3 vehicles, in fact the Highway Chauffeur vehicles are considered able to perform the lane change, especially when driving through a traffic jam [15]. The take-over on the Highway Chauffeur vehicles on the closing lane is imposed so to avoid the software processing step in which the vehicle alone must recognize the roadworks, interpret the horizontal and vertical signage and compute a lane change consequently. This software processing step is still judged critical for an L3 vehicle [1] and it represents the step that differentiates the Highway Chauffeur vehicles driving on the closing lane from the ones driving on the open lane. Moreover, the cooperative message broadcasts the suggested speed limits $(110 \mathrm{~km} / \mathrm{h})$ way ahead the roadworks in both Jointed scenarios, allowing the Highway Chauffeur vehicle to lower their speed in advance, limiting their fuel consumption and having a positive impact on the whole approaching traffic flow for higher market penetrations, as it will be shown in the following sections.

\subsection{Simulated scenarios}

To assess the impacts of the jointed implementation of the C-ITS Use Case and of L3 vehicles, three main scenarios where defined: Jointed scenario 1 (696 m), Jointed scenario $2(1500 \mathrm{~m})$ and No C-ITS scenario. The formers see both the C-ITS message broadcasted but at different distances while in the No C-ITS scenario the L3 vehicles react to the vertical signages as explained above. All these scenarios consider a percentage of heavy vehicles and of commercial vehicles among the traffic flow as recorded on the A22 in the days used to calibrate the model. These vehicles are not equipped for V2X reception in the simulations.

The No C-ITS scenario is the one that sees the hypothesized percentages of market penetration for Highway Chauffeur vehicles but no C-ITS messages. Thus, the L3 vehicles on both lanes should perform the take-over maneuver $696 \mathrm{~m}$ upstream the lane closure. For this scenario the research hypothesis is the following: Without the C-ITS message, the take-over transition should happen on both lanes and for higher market penetrations could delay the lane changes right before the roadworks, decreasing the overall traffic efficiency, even when compared to the baseline scenario with no HC vehicles.

The two Jointed Scenarios include different percentages of market penetration for the Highway Chauffeur vehicles among the light traffic flow $(10 \%, 33 \%, 50 \%$, $66 \%, 80 \%, 100 \%)$. The high number of scenarios related to Market Penetration reflects the aim of the authors to frame the impacts and possible criticalities arising for different time horizons. In fact, it is acknowledged that connected and autonomous vehicles will gradually enter the market and that a long period of transition and coexistence will be faced [16].

As mentioned above, the message about the closure of a lane is received $696 \mathrm{~m}$ or $1500 \mathrm{~m}$ upstream the roadworks. In the first case (Jointed Scenario 1) the potential benefits may arise by the knowledge of the roadworks layout that could allow the L3 vehicles on the open lane to keep driving in automated mode (with all the resulting benefits for Traffic Efficiency, as reported in [1]). The research hypothesis for this scenario is the following: The cooperative message should allow some $H C$ vehicles to keep driving in automated mode, reducing the overall number of take-over maneuver and fostering the remaining ones. This in turn should improve Traffic Efficiency thanks to the perfect speed compliance and reduced headway of the $H C$ vehicles.

In Jointed Scenario 2, as implementation logic the following was adopted: the L3 vehicles on the open lane would keep driving in L3 mode while the L3 vehicles on the closing lane would perform a take-over in advance, the human driver would perform the lane change and these vehicles would take advantage of the increased distance from the roadworks to re-engage L3 driving on the open lane. This last maneuver is also called an upward transition. The research hypothesis for this scenario is the following: The cooperative message should allow CAVs on the closing lane to perform the take-over transition and the lane change in advance, in order to resume automated driving before reaching the roadworks. 


\subsection{COM Interface - modeling a take-over transition in PTV VISSIM}

One of the main synergies between C-ITS services and (semi-)automated vehicles is certainly a smoother, safer take-over transition performed in advance and with more information (as explained in Section 2). This synergy will have impacts both on Safety, Environment and Traffic Efficiency, to quantify these impacts is still a challenge due to the take-over transition being difficult to reproduce on simulation software. To the knowledge of the authors, no micro-simulation software has yet embedded a tool to reproduce this kind of behavior (the only exception possibly being SUMO [11]) while other means of evaluation don't allow to upscale the impact of the transition on the overall traffic flow (e.g. Driving simulators). Thus, a contribute of this paper is the design of a Python script implementable through the VISSIM COM interface and able to reproduce a take-over transition, defined by the authors on the basis of the bibliographical review. In fact, the behavior of a human driver currently out of the driving loop was hypothesized as it follows.

1 When the Highway Chauffeur passes through the section at which the message is broadcasted, if the vehicle finds itself on the lane that is going to be closed (or the section with vertical signage in case No C-ITS), an alert is displayed to the human driver.

2 Once this alert is triggered, the human driver re-enters the driving loop, reaches for the steering wheels and for the pedals and re-assesses situational awareness about the surrounding traffic. This process lasts between $13.5 \mathrm{~s}$ and $18.5 \mathrm{~s}$, during which the driver is not able to perform a lane change. The defined time range is a little wider than $10 \mathrm{~s}$, defined in literature as the minimum time window for a take-over, because the cooperative message is broadcasted in advance and thus the alert is not safety critical. This means that the alert itself is not intrusive and that the human driver reacts comfortably, just as mentioned in Section 2.

3 As soon as the human driver finds itself in the driving loop again, the first action that she/he tries to accomplish is a lane change, just as it would happen in reality after the reception of the message about an upcoming closure of a lane.

4 After the vehicle is in one of the unhindered lanes, the behavior simulated through VISSIM may return to the one adopted for human driving or to the automated one depending on the simulated scenario. In fact, the developed simulations aim to assess the distance that maximizes the benefits related to the C-ITS Use Case and to L3 driving. When the cooperative message is received at $696 \mathrm{~m}$ from the roadworks the take-over distance allows the vehicle to find itself on the open lane short of both time and space to resume automated driving, thus the human driver keeps on driving. When the message is received at $1500 \mathrm{~m}$ from the roadworks, instead, the take-over maneuver and the lane change are accomplished far in advance, with enough space and time for the vehicle to re-engage automated mode.

It should be highlighted that, since the aim of the paper is to compare what is the best implementation from an operational point of view, the transition in control between the two authorities on-board are modelled only in the time needed for them to be carried out. Namely: for the human driver the time slots needed range between $13,5 \mathrm{~s}$ and $18,5 \mathrm{~s}$ while for the automated system the transition is almost instantaneous since the vehicle prompts the re-engagement option only when the ODD is already recovered (i.e. being on the open lane and not at the roadworks entrance). Moreover, the above descriptions cover the two scenarios involving a C-ITS message in order to explain in the following how the script was designed. The scenario NO C-ITS in which the take-over request is issued on both lanes is described in paragraph 4.1 for the sake of the dissertation.

The Python script contains three functions that are called by VISSIM during the simulation, through the COM interface. These three functions are Initialization, ChangeDrivBehavScript, EndDrivBehavScript The last two are the core of the tool (the full Script can be requested by contacting the corresponding author while an extract of these three function is reported in the Additional file 1). The Additional file 1 reports the functions as designed for the Joint Implementation $1(696 \mathrm{~m})$ scenario, but for Jointed implementation $2(1500 \mathrm{~m})$ only slight adjustments are needed to allow the vehicles to re-engage L3 driving once on the open lane.

The two main functions allow to trigger both the transition from automated driving to the take-over transition and the one from the take-over transition to the traditional human behavior. In fact, ChangeDrivBehavScript takes as inputs the links where the take-over request is displayed, the maximum distance needed for the take-over and the take over time (equal to $13.5 \mathrm{~s}$ ).

As it can be seen from Fig. 2, the Scripts takes as input a single value of take-over time but the stochastic nature of this interval is simulated through the COM interface of VISSIM. 


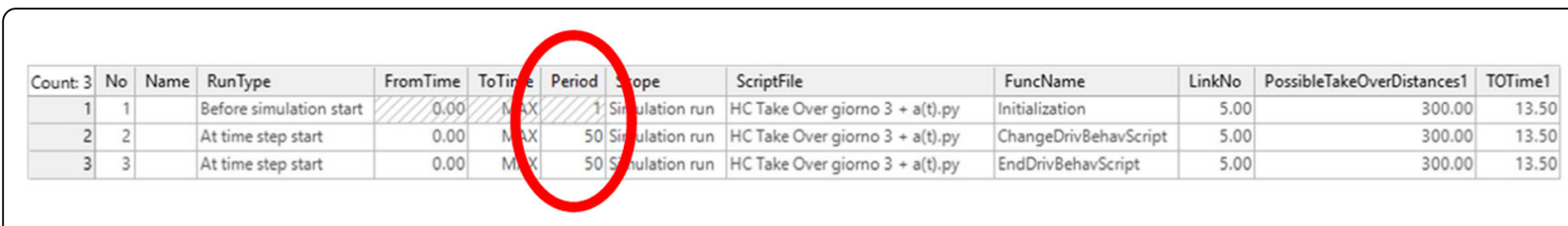

Fig. 2 COM Interface - VISSIM

The micro-simulation software calls both functions every 50 simulation time interval (equal to 5 simulation seconds), which means that every time the script is triggered a different distribution of vehicles has just entered the link number 5 and occupies a certain position within the first $5 \mathrm{~s}$ of driving space. This allows the vehicles to actually display a different and stochastic take-over time that can range between $13.5 \mathrm{~s}$ (the vehicle enters the link just as the script is triggered) and $18.5 \mathrm{~s}$ (the vehicle enters the link the time step after the one in which the script was triggered last time).

Figure 3 shows how, even though the vehicles 1 and 2 enter the link at different time steps, once the script is called at $02: 30^{\prime} 20^{\prime \prime}$, both change vehicle type and the related behavior (displayed as change in color). In fact, as reported within the script, the take-over maneuver is simulated through a vehicle type change, the Highway Chauffeur vehicles are converted into taking-over vehicles and, after a time slot of $13.50 \mathrm{~s}$, they become traditional vehicles driven by the human on-board. It should be highlighted that $02: 30^{\prime} 20^{\prime \prime}$ is just one of the many time steps at which the script is called (it is, in fact, called from the beginning of the simulation every $5^{\prime}$ ) and that Fig. 3 refers to a test network with three lanes and not to the one analyzed in the paper.
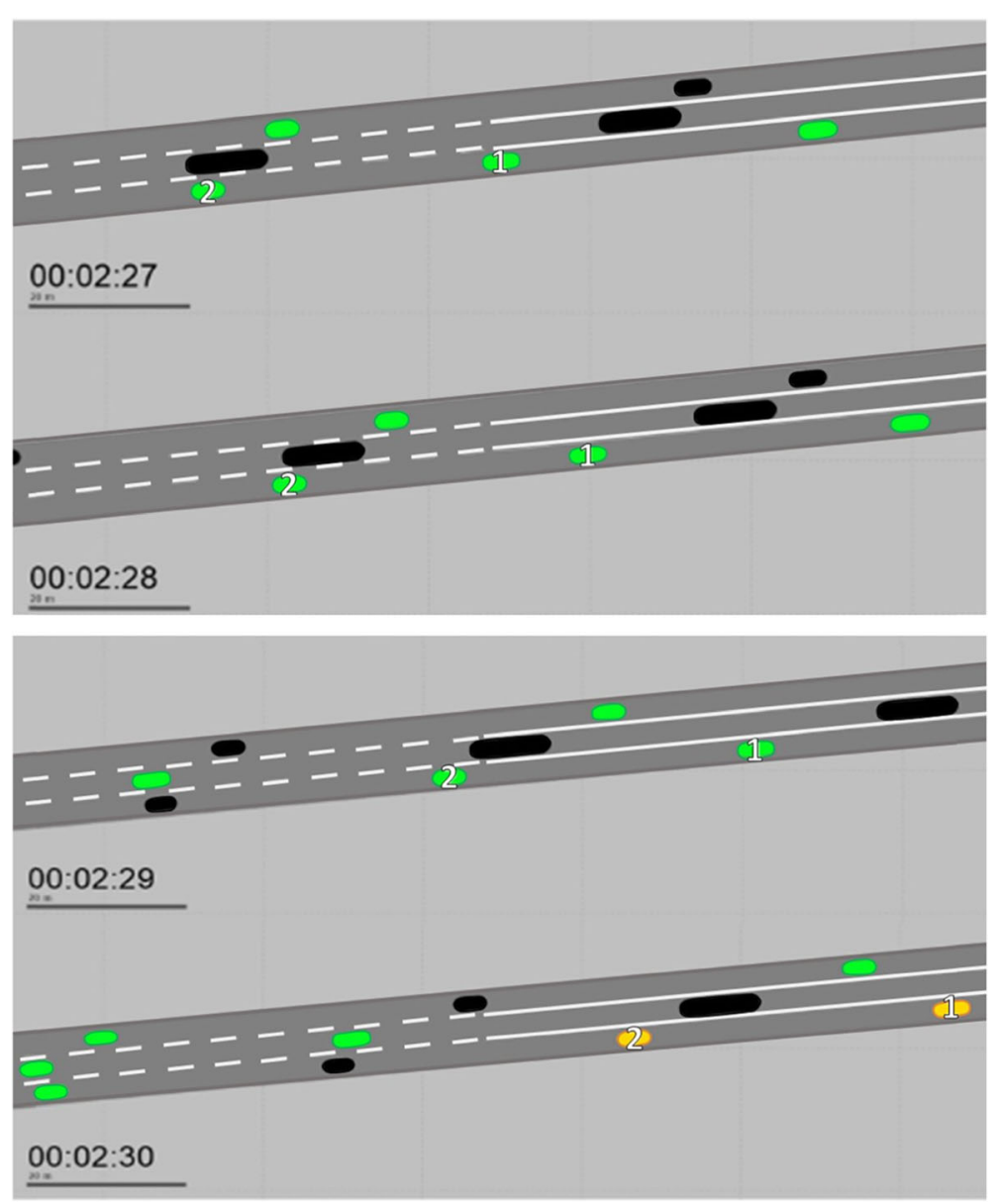

Fig. 3 Stochastic functioning of the script 


\subsection{Driving behavior and modeling parameters}

As mentioned in Section 3, the driving behavior of traditional traffic was calibrated by exploiting traffic data provided by A22 (Autostrada del Brennero). In Fig. 4 a short overview of the outputs of the calibration process is provided:

The calibration and validation process was concluded with an average GEH of 1.55 and values of the Theil's inequality coefficient lower than 0,3 . These results meet the thresholds defined in [17]. The obtained behavioral parameters were then set for traditional vehicles and vehicles after their take-over maneuver. The driving behavior of L3 vehicles was set as the one already compiled within VISSIM and result of the COEXist project [18], keeping a normal level of driving aggressiveness. The headway value was set equal to $1,2 \mathrm{~s}$, with no deviation, which should be kept in mind when analyzing the results in the following Section. The choice of this value is based on the extensive study carried out in $[1,19]$. It should also be pointed out that no traffic data from a congested day was available for the analyzed time period, so it was chosen to reproduce the average traffic flows as recorded in the different scenarios. The volumes are reported in the Fig. 5.

\section{Scenario outputs and results}

Following the approach presented in [19], the KPI chosen to answer the research questions were the speed on each of the lanes through the segment upstream the closure, long about $175 \mathrm{~m}$. These speed measurements should help answer the questions about the Traffic Efficiency for the different scenarios and how the bottleneck performance is affected by the different deployment logics of both the C-ITS (range) and of the $\mathrm{HC}$ vehicles (take-over and lane change). Moreover, the number of vehicles passing through the bottleneck is measured just upstream the closure, on the last point were the two lanes are available to frame both the residual capacity and the distribution of the vehicles on both lanes. Finally, the delay of the traffic on a $240 \mathrm{~m}$ long segment starting $610 \mathrm{~m}$ upstream the closure is measured to monitor the propagation and duration of the disruption.

\subsection{No C-ITS scenario}

The first set of simulations was carried out to obtain what can be considered a baseline for the study, even though this baseline is still strongly projected to a future scenario in which L3 driving is fully operational and available on highways. Still, to obtain the impacts of a jointed implementation between the Highway Chauffeur system and the Use Case Roadworks Warning - Closure of a lane, the first set of results to be analyzed is the one with only L3 driving and a roadworks downstream. To briefly summarize these simulations, the Highway Chauffeur vehicles approaching the lane closure are made aware of the roadworks through signaling in Section 1, $696 \mathrm{~m}$ from the actual closure. At this stage, both lanes can be the one to be closed to the knowledge of the on-board system, therefore on both lanes the takeover maneuver is issued to the drivers through the HMIs. Then the drivers resume control, hindering their lane change for 13.5-18.5 s. Once the control is resumed these vehicles act just like the traditional ones through the simulations. It is clear how the take-over maneuver can have a disrupting effect in this scenario, delaying the re-organizing of a relevant component of the traffic and potentially diminishing the benefits of L3 driving. To verify this hypothesis, the results in Figs. 6 and 7 were obtained and analyzed.

As reported in Fig. 7, the effect at the entrance of the roadworks does not seem to be negative on the closing lane, with a slight increase of driving speed with the increase of Market Penetration during off-peak hours and even a positive effect for high levels of Market Penetration (66-100\%) during the peak hour. A similar effect is recorded also on the open lane, even though the speed improvement during the peak hour is far more limited and arises only for 80 and 100\% Market Penetration. This positive effect at the entrance can be explained by comparing the take-over maneuver on both lanes to a cap, in fact hindering the lane change during the takeover effectively reduces the lane changes of the whole traffic flow on the interested segments. This delay in the lane changes makes so that, when the traffic does not surpass the capacity of the bottleneck a more efficient distribution is obtained and the number of lane changes is kept to a minimum. It should be highlighted that vehicles after the take-over keep a time gap equal to the one kept by traditional light vehicles, therefore there is no additional effect to be considered concerning the speed measurements in Fig. 7. This means that this improvement at the roadworks entrance can be exclusively ascribed to the reduced number of lane changes, here in fact the once-L3 vehicles arrive as traditional ones. Thus, no other factor seems to grant the described benefits rather than the vehicle distribution and speed on the two lanes. Figure 8 gives back a different picture upstream the roadworks entrance instead: no clear trend in delay (and thus in speed) seems to emerge for Market Penetration values ranging between 10 and $66 \%$, with the only real improvement arising for 80 and 100\% and likely bound to the perfect compliance to the speed limit and the reduced gap of the Highway Chauffeur vehicles between Section 3 and 4. Still, it is worth analyzing the mean values of delay over the whole simulation period.

It can be noticed how, even if quite limited, a beneficial effect seems to be bound to the presence of the L3 


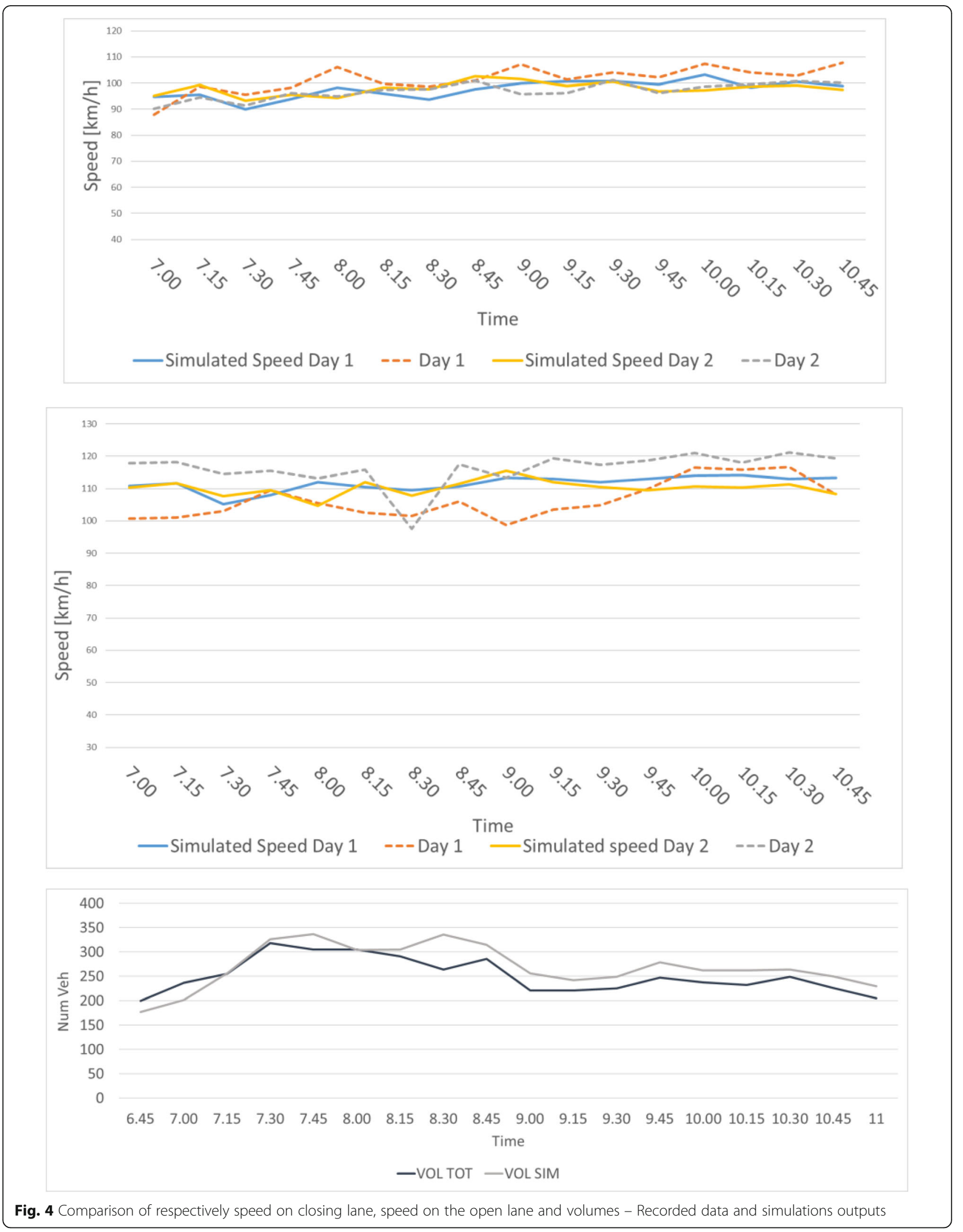




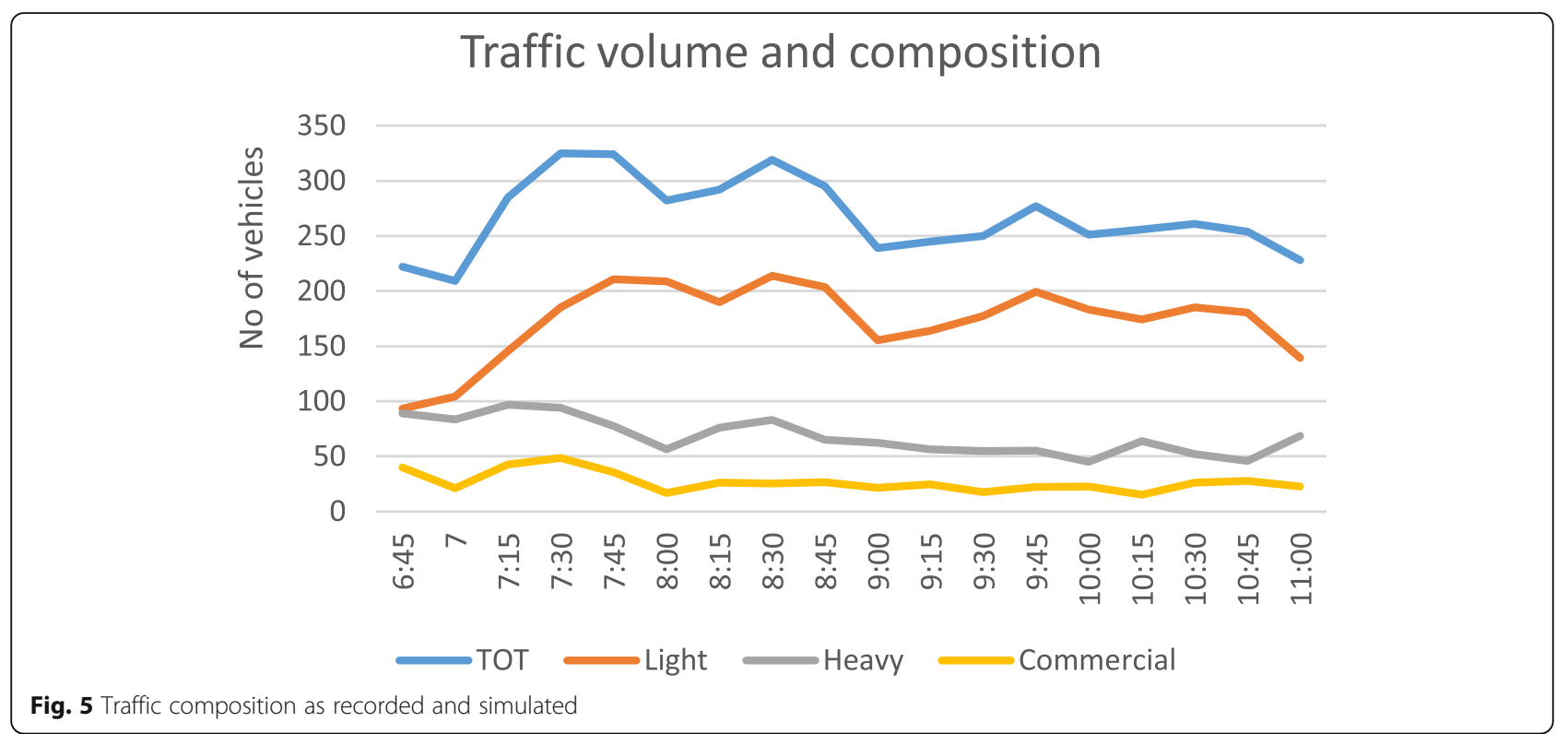

vehicles. It is clearly strongly dependent on the driving behavior simulated and based on the COEXist Project [18], should a more cautious driving regime be implemented by car makers the above results concerning delay could change and even become slightly negative. Still, two factors should be kept in mind:

- The driving logic implemented is considered quite realistic, based on previous work $[1,19]$. In fact, the time gap of $1.2 \mathrm{~s}$ is not a strong hypothesis and neither is the perfect compliance to the speed limits for L3 vehicles (this compliance is not kept by traditional vehicles, as explained in [13]). Moreover, the lane change of the Highway Chauffeur vehicles was tuned to be more conservative than the one performed by the traditional ones (the Min. Headway value is kept 1 for both types while the Safety Distance Reduction Factor is equal to 0.6 for traditional vehicles end to 1 for L3 ones).
- The aim of this paper is not to estimate the impacts related to automated driving on the traffic flow. Instead, the aim is to frame the different impacts on Traffic Efficiency of the three described scenarios bound to the joint implementation, simulated with the same driving behavior for each vehicular class. Therefore, the effects of a certain time gap for L3 vehicles rather than of a certain lane change aggressiveness becomes less relevant for the discussion, being kept consistent through all the scenarios.

- The benefits arising from the presence of L3 vehicles should not overshadow the negative impact on safety that a high number of take-over maneuvers could entail. This paper does not describe these impacts since they can hardly be framed through the exploited tools, still it is worth highlight them to give the reader a complete picture.
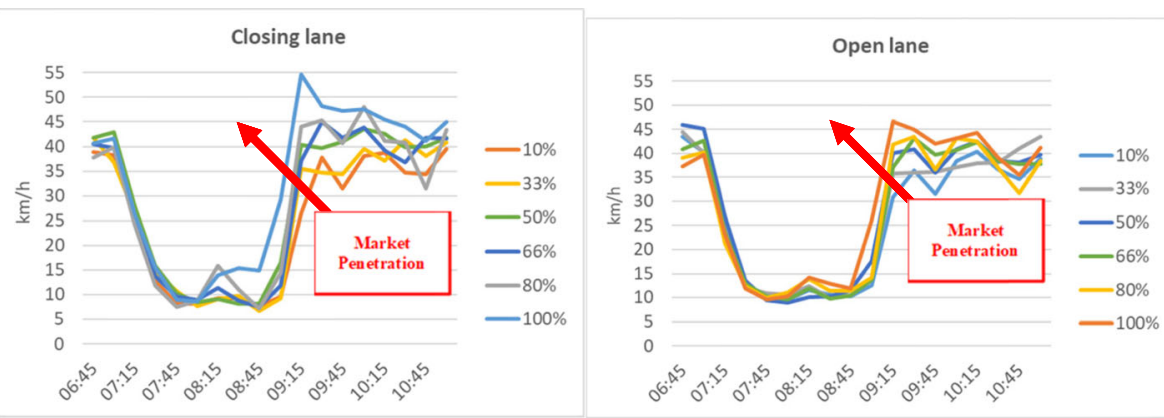

Fig. $6 \mathrm{KPI}$ Speed $[\mathrm{km} / \mathrm{h}]$ at the roadworks entrance 


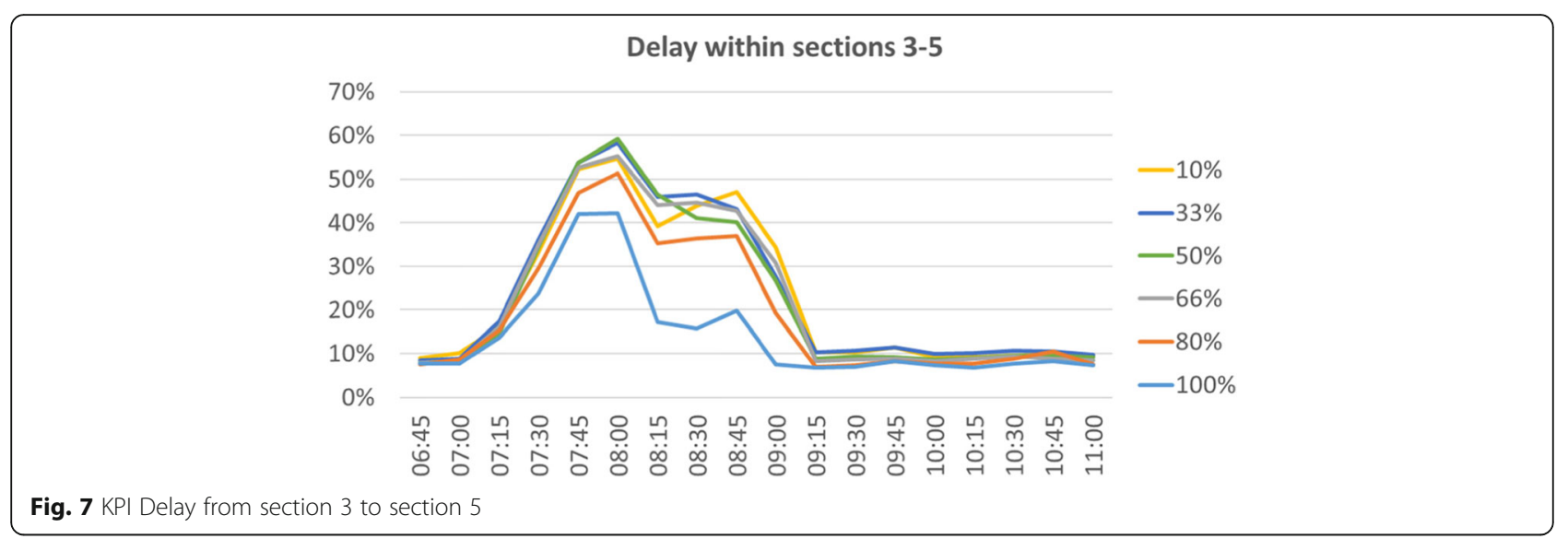

Finally, an interesting result arises from the analysis of the traffic flow on each lane, recorded right upstream the closure and reported in Fig. 8.

It is interesting to notice how the traffic flow does not change with Market Penetration. This is due both to the absence of the C-ITS message and likely also to the "cap effect" mentioned above that hinders rather than fostering the lane changes upstream. In the jointed scenarios, the traffic flows will change with Market Penetration, instead, as it will be discussed.

\subsection{Jointed scenario 1 (696 m)}

In this second implementation scenario, the C-ITS message is used only to replicate physical signaling along the road and upstream the lane closure. Therefore, the broadcasting range is set $696 \mathrm{~m}$ upstream the roadworks and the information sent concerns both the presence of the roadworks and its layout (meaning that the receiving vehicles know in advance which lane will be closed). Following this implementation logic, the Highway Chauffeur vehicles finding themselves on the closing lane when receiving the message issue the take-over request because the time needed for the take-over is hardly compatible with the search for a gap to perform a lane change, since most of the remaining distance is to be used for a safe and smooth take-over. On the other hand, L3 vehicles that are driving on the open lane when receiving the message simply avoid performing a lane change to the closing lane and keep on driving with the system engaged, thus maximizing the benefits both for the drivers and for the surrounding traffic (i.e. less take-over maneuvers, still to be considered a safety concern, and smoother driving with perfect compliance to the speed limits and a lower time gap). This way, Jointed Scenario 1 is designed to maximize the benefit for the overall traffic in this scenario. The arising results are reported in Figs. 9 and 10.

The above results give back an outcome bound to the Market Penetration levels. The speed on both lanes at the roadworks entrance, for example, steadily grows as the number of L3 vehicles on the open lane increases. The speed increases both during the peak hour and during the off-peak hours, which makes clear that this

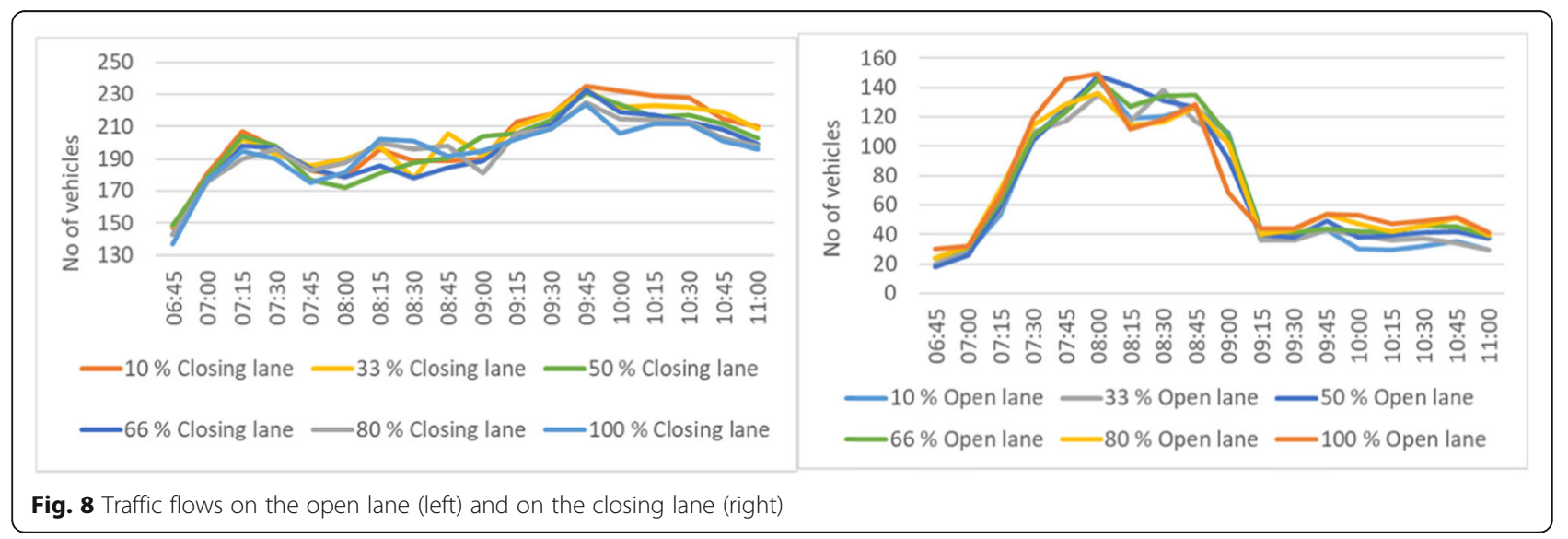




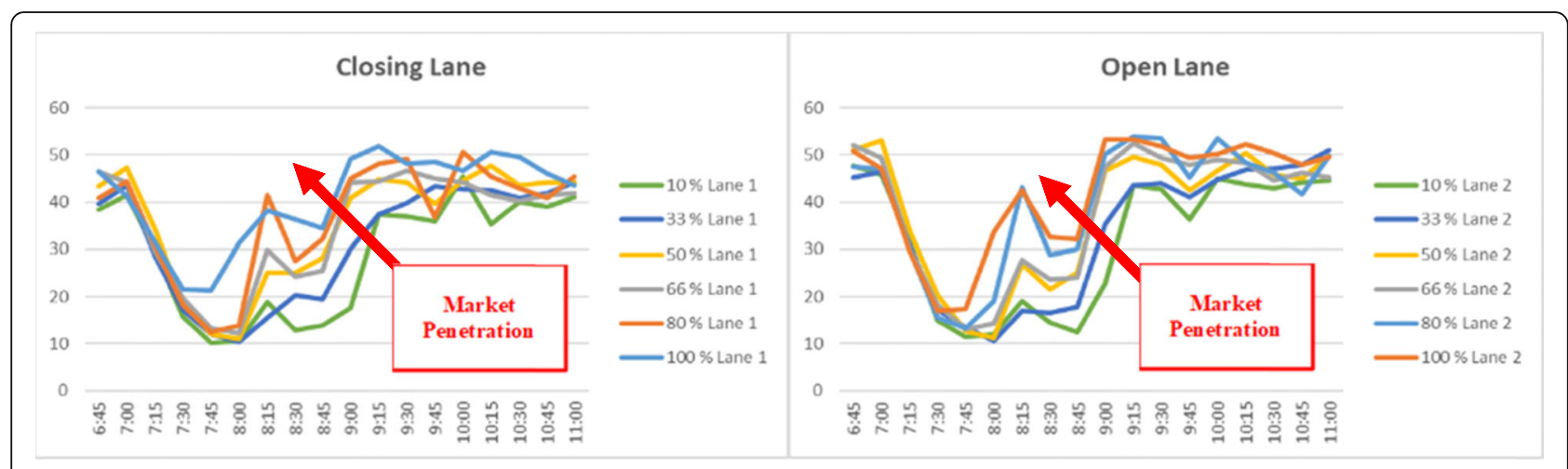

Fig. $9 \mathrm{KPI}$ Speed $[\mathrm{km} / \mathrm{h}]$ at the roadworks entrance

benefit is due to a higher presence of L3 vehicles rather than to a positive effect on the vehicle disposition on the two lanes, on the contrary the number of lane changes is strongly limited in this scenario. Another relevant element is that the described effects propagate upstream, reaching Section 3 as it can be seen from the delay graph in Fig. 11. The C-ITS allows a high number of L3 vehicles to keep on driving without re-engaging the human driver, this high number in return improves travel times and speeds for the overall traffic flow.

Finally, the effect of the C-ITS message that limits a fair share of vehicles on the open lane from the first vertical signage can be observed in the graphs about traffic flows on the two lanes reported in Fig. 11.

\subsection{Jointed scenario $2(1500 \mathrm{~m})$}

This last scenario reflects what, based on the previous work of the authors $[1,19]$, seems to be the Highway Chauffeur operational logic that maximizes the benefits related to Traffic Efficiency for the overall traffic flow. In fact, the message broadcasted upstream the actual vertical signage allows the L3 vehicles on the closing lane to perform the take-over maneuver and then, enjoying the increased distance, to perform a manual lane change (namely, performed by the human driver). Then, being on the open lane but not yet within a critical zone such as the roadworks one nor near the lane closure, the vehicle resumes L3 mode and keep on driving as an automated vehicle.

The simulation results confirm that no vehicles arrive at the lane closure while in take-over mode with the timing hypothesized in Section 3. This strategy allows the Highway Chauffeur vehicles to return to manual driving for the minimum number of kilometers, thus maximizing the benefits related to automated driving and reported in the previous scenario (e.g. reduced time gaps, perfect compliance, etc.). Clearly, L3 vehicles on

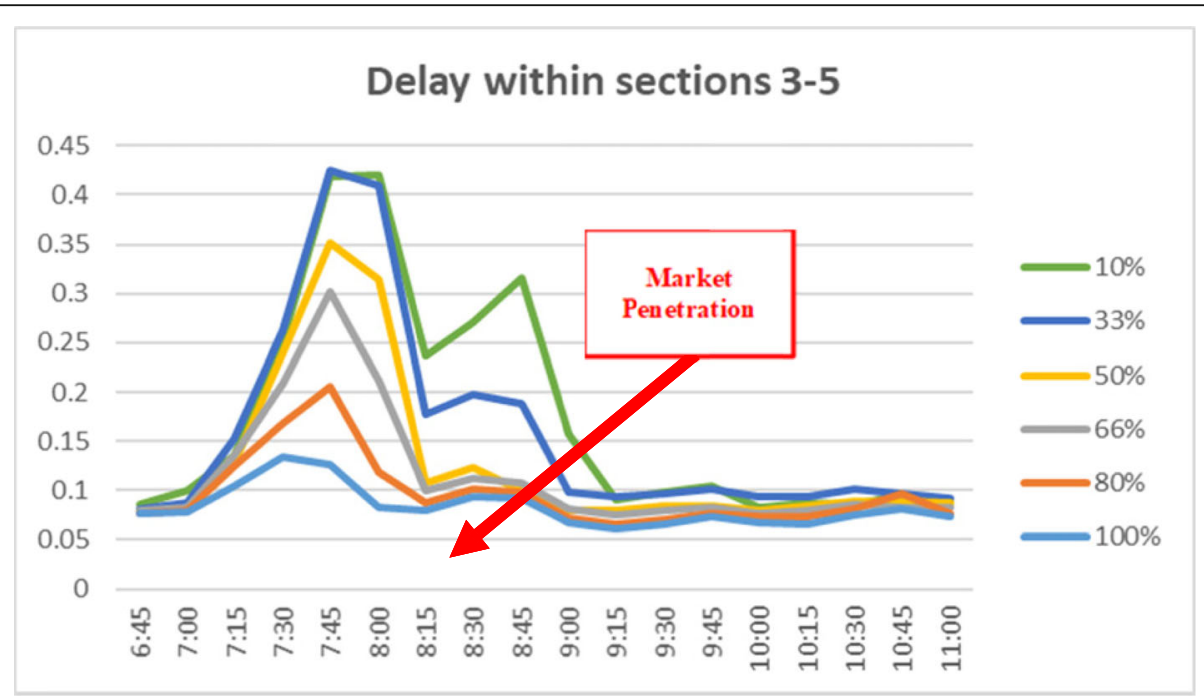

Fig. 10 KPI Delay from section 3 to section 5 




Fig. 11 Traffic flows on the open lane (left) and on the closing lane (right)

the open lane $1500 \mathrm{~m}$ upstream the lane closure behave as in the previous scenario and do not perform a lane change on the closing lane. The obtained results are reported in Fig. 12 and in Fig. 13.

The achieved effects are similar to the ones of Jointed Scenario 1, even though they seem steadier (especially in the off-peak hours) and higher in magnitude (the speed within the peak-hour reaches non-congested values for high Market Penetrations). It was to be expected to have results similar in their trending to the preceding scenario, in fact both are heavily dependent on an implementation of automated driving that is beneficial for the traffic flow. The contribute granted by the C-ITS simply increases the number of vehicles able to pass through the roadworks in L3 driving (on the open lane in the Jointed Scenario 1 and on both lanes in Jointed Scenario 2). Still, a result that should be highlighted in its worth is that the re-organizing of this class of vehicles does not disrupt the overall traffic flow, even for high Market Penetrations. This is likely due to the presence of the overtaking ban for heavy vehicles and to the geometrical features of A22 motorway, that sometimes can force a certain share of vehicles on the closing lane. This in turn guarantees that the infrastructure is not under-utilized by having all the vehicles only on one lane, which is proved by the certain shares of vehicles that still arrives to the lane closure on the closing lane, as can be seen in Fig. 14 (right).

\section{Scenario comparison}

The above results have their own worth in depicting the effects that L3 driving and C-ITS could have on a roadwork bottleneck. Still, the analysis would not be complete without a critical comparison to derive what is the net benefit (or negative impact) in deploying one implementation logic rather than another. The results that the paper aims to obtain in this Section should support both Road Operators in defining the range of the message and the allowed maneuvers and the Car Maker in defining the driving logics to implement within the driving software as well as the distance at which the message should be broadcasted on the HMI to guarantee a safe and smooth take-over transition. On the basis of what is stated above, the obtained KPIs where compared between scenarios for each Market Penetration level.

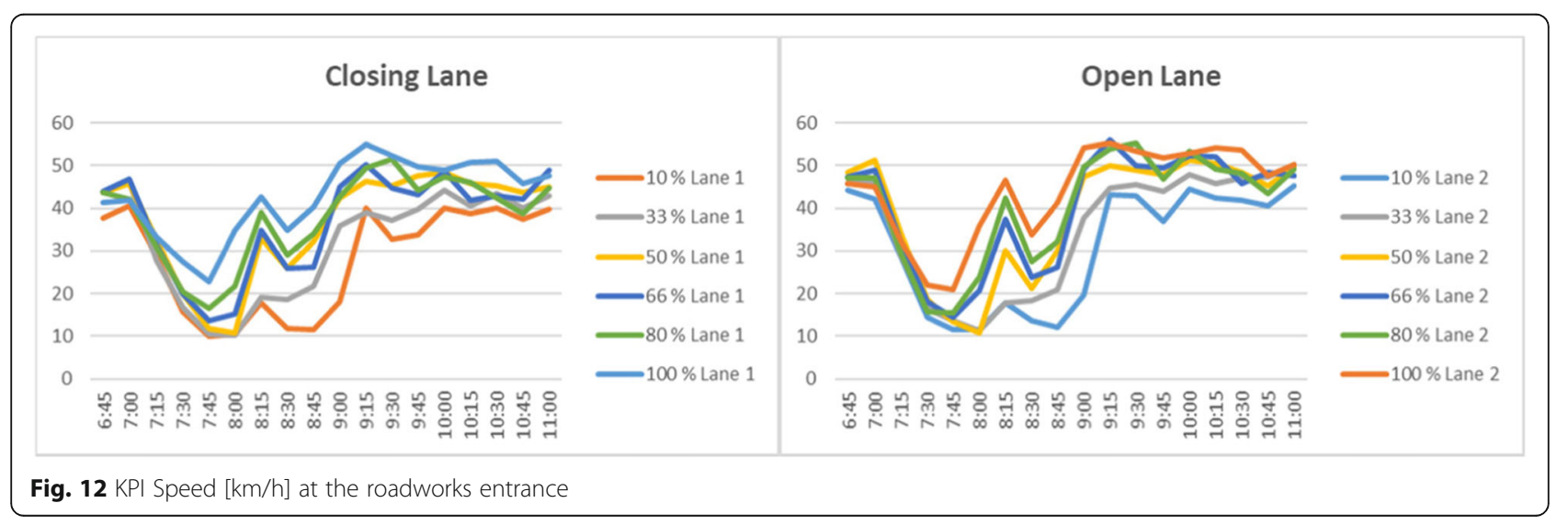




\section{Delay within sections 3-5}

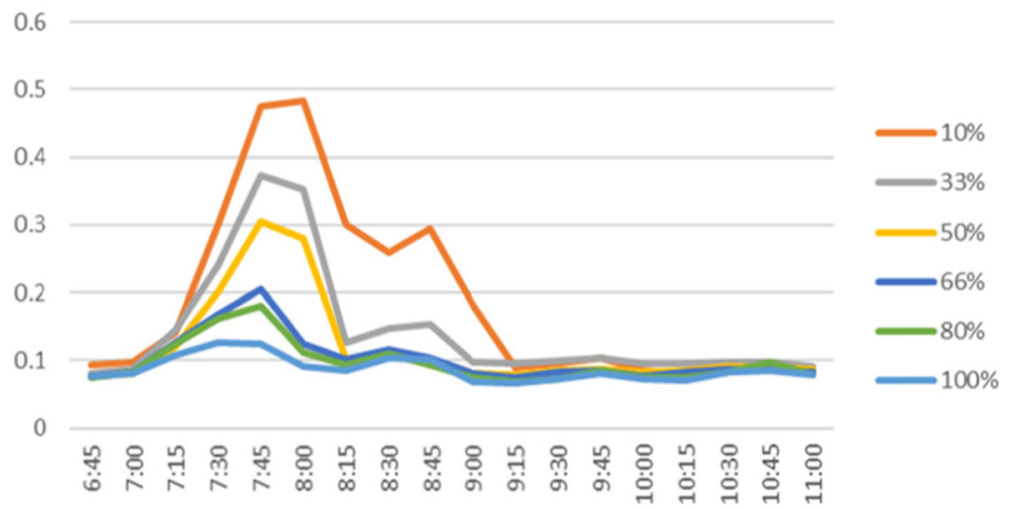

Fig. $13 \mathrm{KPI}$ Delay from section 3 to section 5

In the graphs reported in Fig. 15, the speed right upstream the lane closure on the closing lane is compared. The graphs report the speed value $[\mathrm{km} / \mathrm{h}]$ on the $y$ axis, the time on the $\mathrm{x}$ axis and the Market Penetration on the $\mathrm{z}$ axis.

From Fig. 15 it can be stated that the presence of the C-ITS message, and therefore of a certain share of L3 vehicles driving following the implementation logics detailed in Section 4, improves the driving speed at the roadworks entrance both during the peak hour and during the off-peak. It is relevant to note also that this trend perfectly follows the Market Penetration one, as can be clearly noted by the gray and yellow peak during 8:30 AM. Another important reason causing this effect is the reduced number of vehicles performing the lane change right at the roadworks entrance (decreased through the C-ITS). In Fig. 16, the same KPI for the open lane is provided.

A similar effect arises on the fast lane, also fostered by the reduced number of vehicles performing a lane change right at the roadworks entrance. It is also important to highlight how the benefits related to the speed increase steadily of $10 \mathrm{~km} / \mathrm{h}$ in the off-peak hours for every level of Market Penetration: this $\Delta$ reaches values of $30 \mathrm{~km} / \mathrm{h}$ during the peak hour for high levels of market penetrations and values of $20 \mathrm{~km} / \mathrm{h}$ starting yet from 50\% Market Penetration in the Jointed Scenario 2 (circled areas in Fig. 16).

Finally, the average delays recorded upstream for each Market Penetration in the three scenarios are reported in Table 1 to give an overview of the potential arising benefits. These results were widely discussed in Section 4 but it is worth reporting them together to show how clear is the trend arising with Market Penetration of both L3 vehicles and of the C-ITS system. It is also worth highlighting how Jointed Scenario 1 and 2 are comparable in their effects on this KPI while Jointed Scenario 2 outperform Jointed Scenario 1 on the KPIs recorded at the roadworks entrance.

It should also be reported that the maximum net benefits on delay (around $9 \div 10 \%$ ) is relevantly higher than the one achieved only through connected vehicles which is equal to $3.24 \%$ [20]. In [20] the authors report the

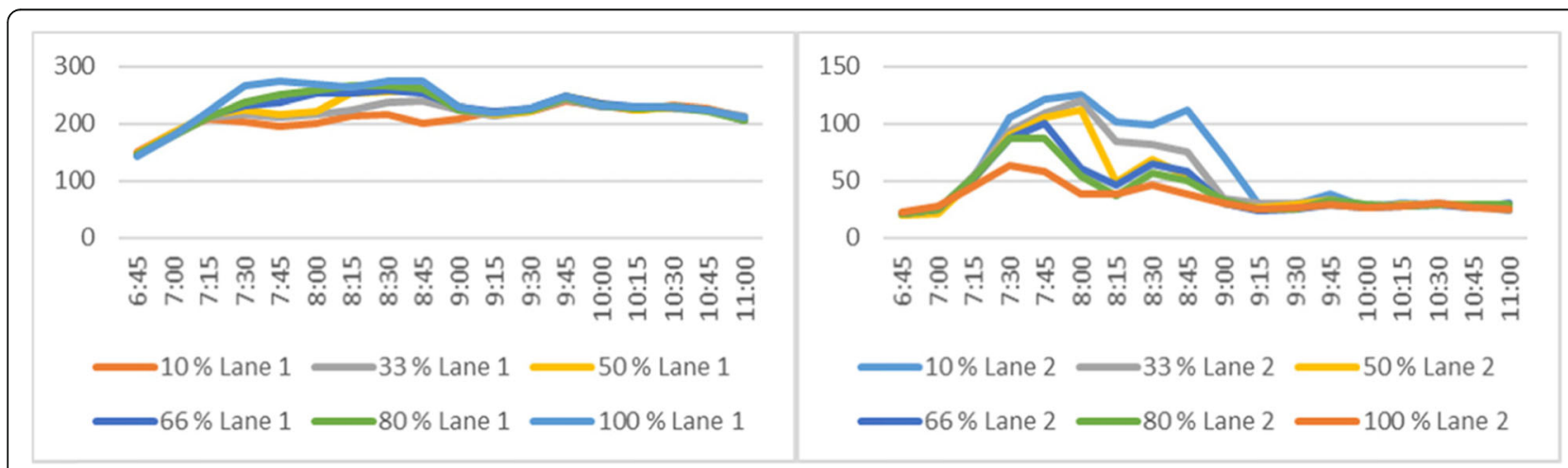

Fig. 14 Traffic flows on the open lane (left) and on the closing lane (right) 

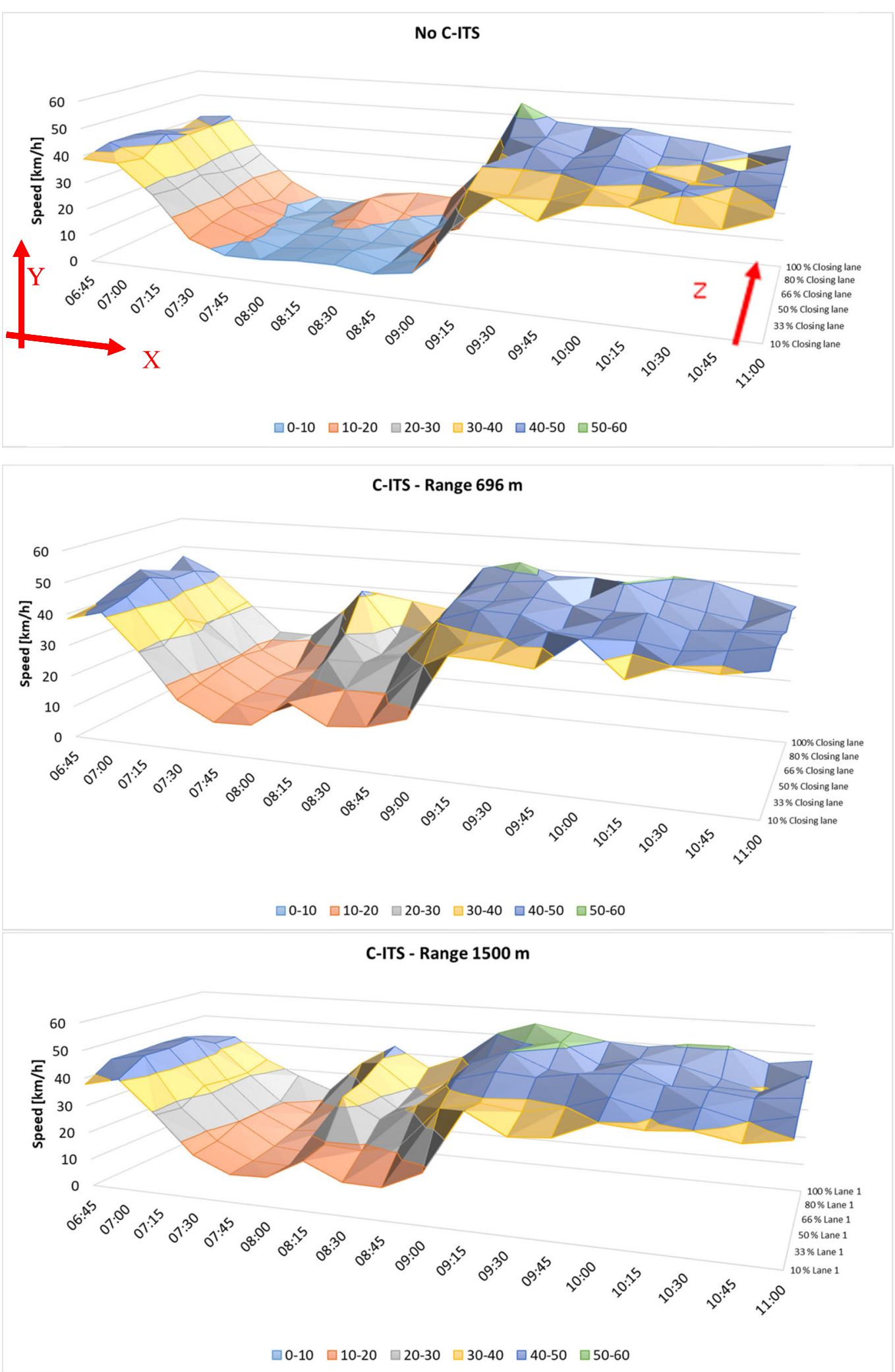

Fig. 15 Scenario comparison - Speed on the closing lane 

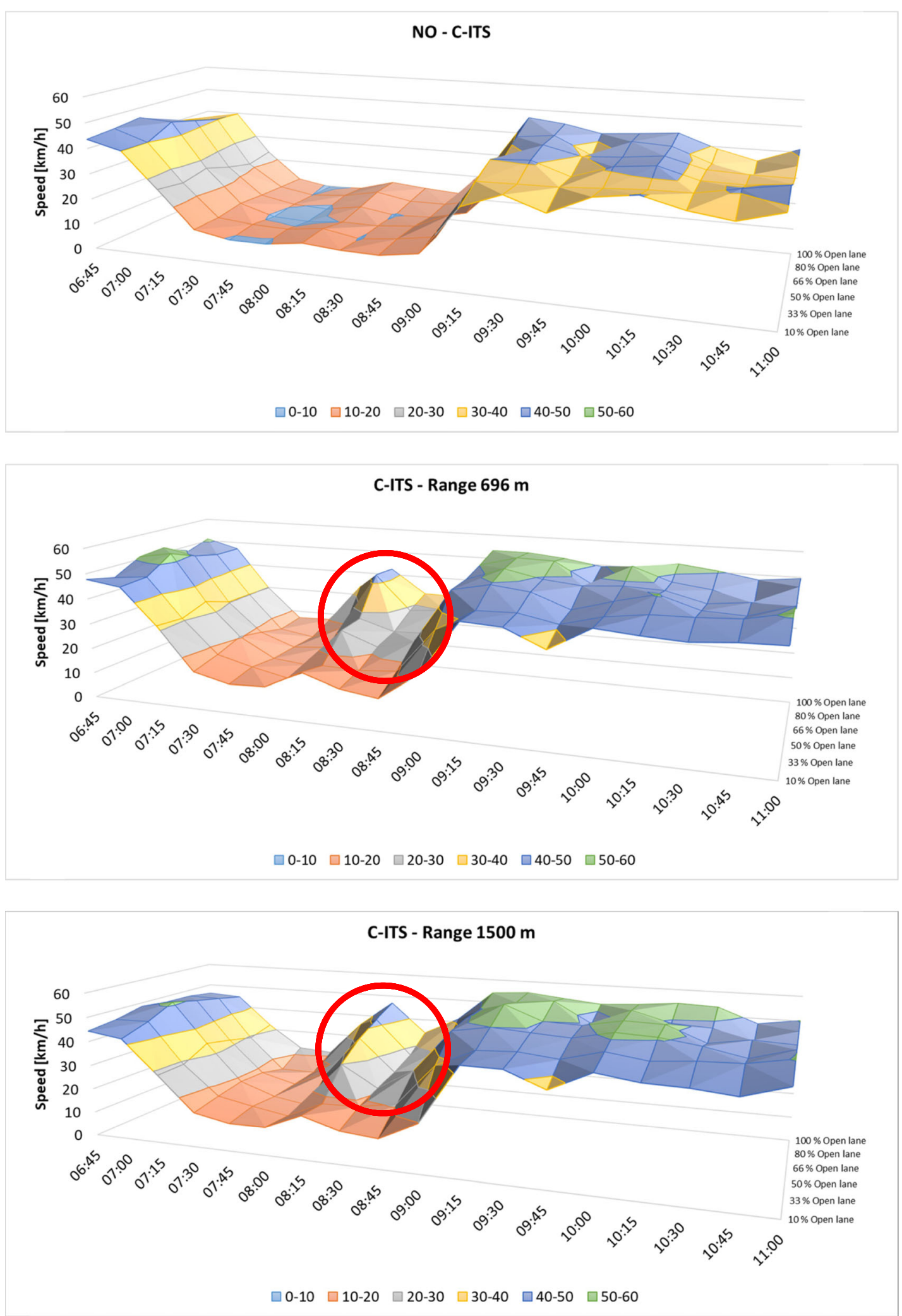

Fig. 16 Scenario comparison - Speed on the open lane 
Table 1 Average delays within sections 3 and 5

\begin{tabular}{lllllll}
\hline & $\mathbf{1 0} \%$ & $\mathbf{3 3} \%$ & $\mathbf{5 0} \%$ & $\mathbf{6 6 \%}$ & $\mathbf{8 0} \%$ & $\mathbf{1 0 0} \%$ \\
\hline NO C-ITS & $23.26 \%$ & $23.84 \%$ & $22.54 \%$ & $22.57 \%$ & $19.54 \%$ & $14.25 \%$ \\
$\mathbf{6 9 6} \mathbf{m}$ & $17.34 \%$ & $15.81 \%$ & $12.65 \%$ & $11.47 \%$ & $9.69 \%$ & $8.27 \%$ \\
$\mathbf{1 5 0 0} \mathbf{m}$ & $18.61 \%$ & $14.36 \%$ & $11.93 \%$ & $10.26 \%$ & $9.68 \%$ & $8.75 \%$ \\
\hline
\end{tabular}

effects on the same KPI due just to the implementation of connected vehicles and the C-ITS message. This comparison can be performed since both works focus on the A22 network, exploit the same traffic data and analyses the same layout, being part of the set of results obtained within the C-Roads Italy project. Thus, an approximate value of the $\Delta$ in benefits/downsides due to L3 driving and the take-over maneuver can be drawn by comparing the two sets of results. Further details can be found in [20]. In fact, this paper is one of the research outputs of the C-Roads Italy project together with the C-ITS assessment [20] and the development of another more complex script aimed at simulating the Truck Platooning system and its interaction with C-ITS services [21-23]
Finally, a really interesting result arises from the analysis of the number of lane change maneuvers, as reported in the Fig. 17. As it can be seen, in the end it is not the C-ITS message that impacts on a reduction of these maneuvers, since the presence of the taking-over vehicles weights much more. This is most likely due, as hypothesized, to the L3 vehicles forced to not change lane in a critical segment of the upstream segment. This rigidity imposed to the traffic influences also the other vehicles and this influence increases with the number of L3 vehicles, as it can be observed in the results concerning the No C-ITS Scenario (in which the number of lane changes strongly decreases with the increase of L3 vehicles).

It should be observed that the number of "to the left" lane changes is higher since part of the traffic has to leave the closing lane in every scenario. The impact of the C-ITS message is null in this dynamic, regardless of the broadcasting distance, since the L3 vehicles on the closing lane receiving the message only perform the lane change once (both at $1500 \mathrm{~m}$ and at $696 \mathrm{~m}$ ) as they

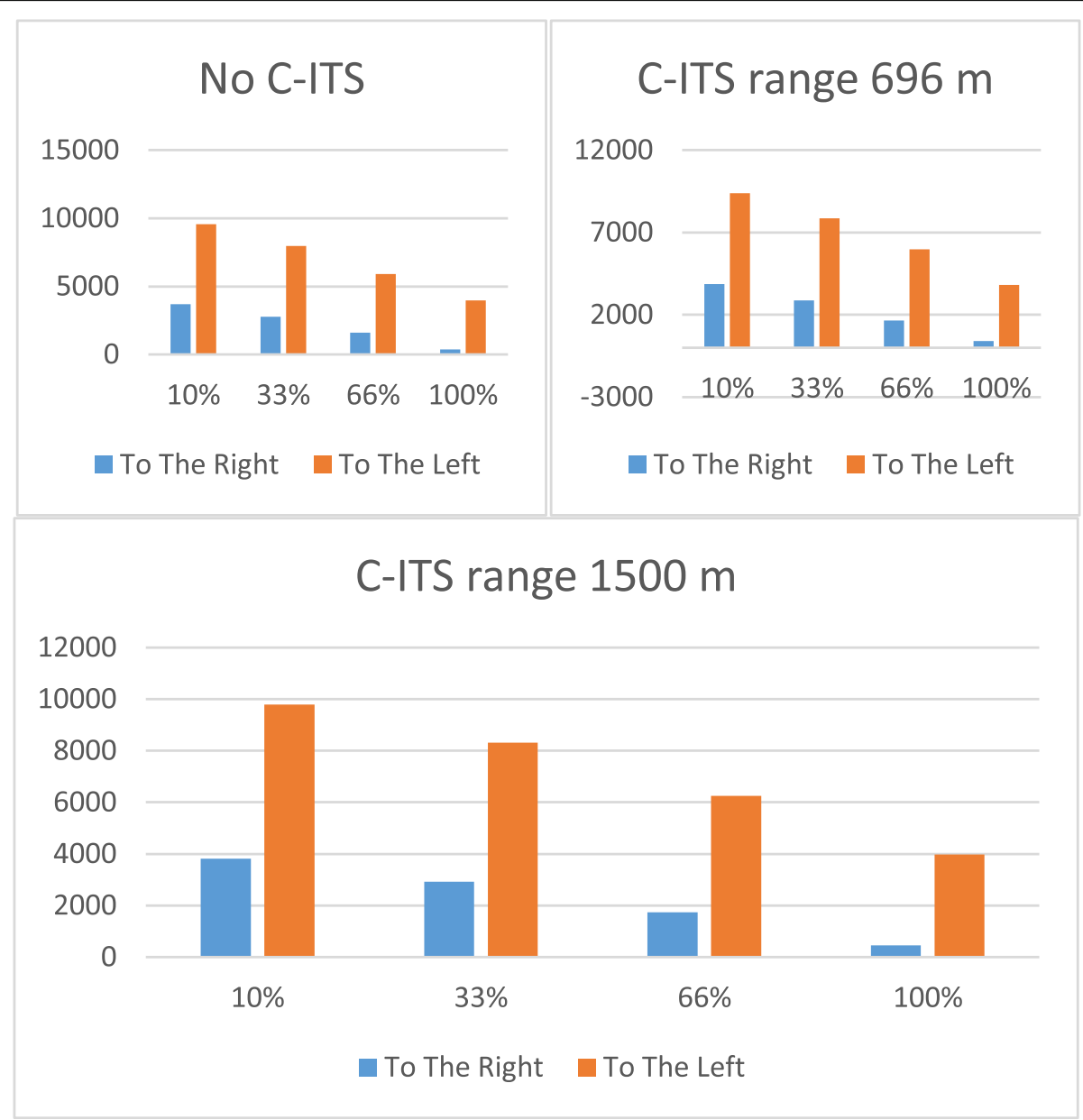

Fig. 17 Number of lane changes 
should to enter the roadworks. It is interesting to note though that also without C-ITS the L3 vehicles end up doing a single lane change from the closing lane to the open one; this is due to the fact that they are forced to avoid lane changes during the take-over maneuver, thus having only the few dozen meters before the roadworks to adapt their position on the road. As a future research direction, a more focused study on the exact position of these lane changes and how it varies by imposing the take-over at different points upstream the roadworks would be valuable in framing where this positive effect arising from the take-overs loses its strength and leaves room to the C-ITS messages to actually influence the number of lane changes. Also, better understanding about how connected human driven vehicles would enter this dynamic depending on different broadcasting range should be further analyzed.

\section{Conclusion}

The paper aim was to evaluate the effects of the jointed implementation both on the Highway Chauffeur vehicles and on the surrounding traffic. To do so, the three research questions about Traffic Efficiency reported in section 1 were answered and the following results obtained. These were:

- Does carrying the take-over maneuver in advance to the roadworks vertical signage foster an enhancement in Traffic Efficiency across the bottleneck section?

- If the C-ITS broadcasts what is the closed lane and allows vehicles on the open one to keep on driving in the automated mode does the overall traffic flow benefits from that in terms of Traffic Efficiency?

- What are the impacts on the upstream section in terms of delay?

While answering the first research question, it is possible to state that triggering the take-over maneuver in advance $(1500 \mathrm{~m}$ rather than $696 \mathrm{~m})$ foster the bottleneck efficiency, which is reflected by a higher speed at the roadworks entrance. In fact, a value between 30 and $40 \mathrm{~km} / \mathrm{h}$ during peak hour is achieved only for Market Penetrations of 80 and 100\% for 696 $\mathrm{m}$ while this range is reached already at $50 \%$ Market Penetration for $1500 \mathrm{~m}$ ). It should be highlighted that the distance at which the C-ITS is transmitted is relevant mainly in the measure it defines if the L3 vehicles on the closing lane are able to resume their automated driving before entering the roadworks. Future researches should investigate what would be the exact safe minimum distance for Jointed Scenario 2, focusing also on Safety.
Table 2 Mean delay values over the simulation period

\begin{tabular}{lllllll}
\hline MP & $\mathbf{1 0 \%}$ & $\mathbf{3 3 \%}$ & $\mathbf{5 0 \%}$ & $\mathbf{6 6 \%}$ & $\mathbf{8 0} \%$ & $\mathbf{1 0 0 \%}$ \\
\hline Average Delay & $23.26 \%$ & $23.84 \%$ & $22.54 \%$ & $22.57 \%$ & $19.54 \%$ & $14.25 \%$ \\
\hline
\end{tabular}

The answer to the second research question lies in the increased share of $\mathrm{HC}$ vehicle able to keep the automated mode engaged on the open lane if the roadworks layout is broadcasted. This share translates into benefits for the whole traffic flow indeed, which are framed in the Jointed Scenario 1. In this scenario, an increase in speed at the bottleneck equal to around $10 \mathrm{~km} / \mathrm{h}$ on both lanes for lower values of Market Penetration (30 $\div 40 \%)$ is recorded. This value grows up to $20 \mathrm{~km} / \mathrm{h}$ on the closing lane and to $30 \mathrm{~km} / \mathrm{h}$ on the fast lane for higher Market Penetration values. Jointed Scenario 2 is featured by similar results, even though the Market Penetration needed to achieve them is lower by $10 \%(20 \div 30 \%$ is enough to achieve $\Delta$ similar to the ones arising with a broadcasting range of $696 \mathrm{~m}$ ). Moreover, a positive effect on the speed upstream the roadworks is achieved for ranges of both $696 \mathrm{~m}$ and $1500 \mathrm{~m}$, as reported in Table 2 and further explained while answering the last research question.

To answer the third research question, the delay on the upstream segment for the three scenarios should be analyzed indeed. In fact, to trigger the take-over maneuver in advance $(1500 \mathrm{~m})$ seems to have limited effects when compared to the minimum distance of $696 \mathrm{~m}$. By broadcasting the roadworks layout at this distance rather than exploiting only the vertical signage seems to entail high benefits instead. These considerations are based on the delay results summarized in Table 2 . As it can be observed by limiting the number of take-overs, the C-ITS message generates a net benefit of $6 \%$ on the delay upstream the roadworks. As a future research direction, this result should be analyzed on a more congested network to frame the potential benefits of L3 vehicles on queue formation and propagation.

The paper frames the overall traffic dynamics given a certain driving behavior of the Highway Chauffeur vehicles, future works could investigate how the increased share of L3 vehicle would impact roadworks with different driving logics (e.g. really aggressive automated driving, platooning or even very cautious behavior). Moreover, the simulated behavior during take-over could be validated through field tests and tests in virtual reality to improve the results. Still, in this paper the effects of the C-ITS Use Case Roadworks Warning - Closure of a lane seems to be framed, namely how much the message can foster the effect of conditionally automated driving on the surrounding traffic and on the bottleneck. 


\section{Supplementary Information}

The online version contains supplementary material available at https://doi. org/10.1186/s12544-020-00457-z.

\section{Additional file 1.}

\section{Acknowledgements}

Not applicable.

\section{Authors' contributions}

Conceptualization, Agriesti S., Ponti M., Marchionni G., Gandini P. Methodology, Agriesti S., Gandini P. Software, Agriesti S., Ponti M. Validation, Marchionni G., Ponti M., Gandini P. Investigation, Agriesti S., Gandini P., Marchionni G. Resources, Agriesti S., Ponti M. Data curation, Agriesti S. Supervision, Ponti M., Marchionni G. Writing, Agriesti S. The authors read and approved the final manuscript

\section{Funding}

Not applicable.

\section{Availability of data and materials}

The traffic data used to calibrate the model cannot be provided in its raw form, being A22 the lawfully owner of these data. The script cannot be provided on a repository Open Access as long as the research is not published but it will be made available by the corresponding author upon request.

\section{Competing interests}

The authors declare no conflict of interest.

\section{Author details}

'Built Environment - Aalto Yliopisto, Otakaari 4, 02150 Espoo, Finland. ${ }^{2}$ Mobility and Transport Laboratory - Dipartimento di Design, Politecnico di Milano, Via Durando 38/A, 20158 Milan, Italy.

Received: 13 February 2020 Accepted: 17 November 2020

Published online: 04 January 2021

\section{References}

1. Studer, L., Agriesti, S., Ponti, M., Gandini, P., Marchionni, G., Borghetti, F., ... Bruglieri, M. (2018). "Evaluation approach for a combined implementation of Day 1 C-ITS and Highway Chauffeur - v.1.0.," Ex-Ante Evaluation Report, CRoads Italy Platform.

2. Agriesti, S., Studer, L., Gandini, P., Marchionni, G., \& Ponti, M. (2019). Safety on the Italian highways, impacts of the highway chauffeur system. In Smart innovation, Systems and Technologies, (vol. 149, pp. 57-73).

3. C-Roads Platform, Working Group 3 evaluation and assessment. Evaluation and Assessment Plan - Final Version, May 2018

4. Barnard, Y., Chen, H., Koskinen, S., Innamaa, S., Gellerman, H., Svanberg, E., ... Brizzolara, D. (2017). FOT-Net Data Field Operational Test Networking and Data Sharing Support D5.4.

5. Studer, L., Agriesti, S., Gandini, P., Marchionni, G., \& Ponti, M. (2018). Highway chauffeur: State of the art and future evaluations: Implementation scenarios and impact assessment. In International conference of electrical and electronic Technologies for Automotive.

6. Zeeb, K., Buchner, A., \& Schrauf, M. (2015). What determines the take-over time? An integrated model approach of driver take-over after automated driving. In Accident Analysis \& Prevention, (vol. 78, pp. 212-221).

7. Son, J., \& Park, M. (2017). Situation Awareness and Transitions in Highly Automated Driving: A Framework and Mini Review. Journal of Ergonomics, 7(5), pp. 1-6

8. Melcher, V., Rauh, S., Diederichs, F., Widlroither, H., \& Bauer, W. (2015). Takeover requests for automated driving. Procedia Manufacturing, 3, 2867-2873.

9. Merat, N., Jamson, A. H., Lai, F. C. H., Daly, M., \& Carsten, O. M. J. (2014). Transition to manual: Driver behaviour when resuming control from a highly automated vehicle. Transportation Research Part F: Traffic Psychology and Behaviour, 27, 274-282.

10. Forster, Y., Naujoks, F., Neukum, A., \& Huestegge, L. (2017). Driver compliance to take-over requests with different auditory outputs in conditional automation. Accident Analysis \& Prevention, 109, 18-28.
11. Lücken, L., Mintsis, E., Porfyri, K. N., Alms, R., Flötteröd, Y., \& Koutras, D. (2019). From Automated to Manual - Modeling Control Transitions with SUMO. In EPiC Series in Computing, (vol. 62, pp. 124-144).

12. C-Roads Platform, "Common C-ITS Service Definitions", Working Group 2 Technical Aspects, Taskforce 2 Service Harmonization, version 1.3, 17.09. 2018. https://itscorridor.mett.nl/c-its+corridor/Achtergronddocumenten/ handlerdownloadfiles.ashx?idnv=1514252.

13. S. Agriesti, P. Gandini, G. Marchionni, M. Ponti, L. Studer, Q. Xiaobo, Roadworks Warning - Closure of a Lane, the impact of C-ITS messages, (in press)

14. Disciplinare per l'installazione, conduzione e rimozione dei cantieri di lavoro sulla rete di autostrade per I'Italia, Autostrade per l'Italia, DCOP/ Coordinamento Viabilità - Edizione giugno 2017

15. ERTRAC WORKING GROUP - Connectivity And Automated Driving, "Automated Driving Roadmap" May 2017. [Online]. Available: http://www. ertrac.org/index.php?page=ertracroadmap. [Accessed: September 2017].

16. Kamal, M. A. S., Ramezani, M., Wu, G., Roncoli, C., Rios-Torres, J., \& Orfila, O (2020). Partially Connected and Automated Traffic Operations in Road Transportation. Editorial in Journal of Advanced Transportation, 2020, 9490586

17. Z.F. Nassrullah, Development of a micro-simulation model for motorway Roadworks with the use of narrow lanes and lane closure schemes, School of Computing Science and Engineering, University of Salford (Manchester UK), 2016

18. COEXist Project, https://www.h2020-coexist.eu/what-is-coexist/ [Online]. Accessed on 07/02/2020.

19. Studer, L., Agriesti, S., Gandini, P., Marchionni, G., \& Ponti, M. (2019). Impact assessment of cooperative and automated vehicles. In M. Lu (Ed.), Cooperative Intelligent Transport Systems: Towards High-Level Automated Driving. London: IET (Institution of Engineering and Technology) ISBN: 978183953-012-8 (Print) / 978-183953-013-5 (eBook).

20. L. Studer, S. Agriesti, P. Gandini, G. Marchionni, M. Ponti, "Highway chauffeur: State of the art and future evaluations : Entation scenarios and impact assessment" in 2018 international conference of electrical and electronic Technologies for automotive, Automotive 2018

21. S. Agriesti, L. Studer, M. Ponti, G. Marchionni, P. Gandini, X. Qu, Roadworks Warning - Closure of a Lane, the impact of C-ITS messages, (in press)

22. Agriesti, S., Gandini, P., Marchionni, G., Paglino, V., Ponti, M., \& Studer, L. (2018). Evaluation approach for a combined implementation of day 1 C-ITS and truck platooning. In IEEE Vehicular Technology Conference, (pp. 1-6).

23. S. Agriesti, P. Gandini, G. Marchionni, M. Ponti, L (2010). Studer, Definition of a Python script for the micro-simulation of the Truck Platooning system, (in press)

\section{Publisher's Note}

Springer Nature remains neutral with regard to jurisdictional claims in published maps and institutional affiliations.

\section{Submit your manuscript to a SpringerOpen ${ }^{\circ}$ journal and benefit from:}

- Convenient online submission

- Rigorous peer review

- Open access: articles freely available online

- High visibility within the field

- Retaining the copyright to your article

Submit your next manuscript at $\boldsymbol{\nabla}$ springeropen.com 